\title{
Caractéristiques de départ et issues chez des patients atteints de COVID-19 hospitalisés dans des unités de soins intensifs à Vancouver (Canada) : série de cas
}

\author{
Anish R. Mitra MD MPH, Nicholas A. Fergusson MSc, Elisa Lloyd-Smith PhD, Andrew Wormsbecker MD, \\ Denise Foster RN, Andrei Karpov MD MHSc, Sarah Crowe NP, Greg Haljan MD, Dean R. Chittock MD MS(Epi), \\ Hussein D. Kanji MD MPH, Mypinder S. Sekhon MD, Donald E.G. Griesdale MD MPH
}

Citation : CMAJ 2020 June 29;192:E694-701. doi: 10.1503/cmaj.200794-f; diffusion hâtive le 27 mai 2020

Voir la version anglaise de l'article ici : www.cmaj.ca/lookup/doi/10.1503/cmaj.200794

\section{RÉSUMÉ}

CONTEXTE : La pandémie de maladie à coronavirus du syndrome respiratoire aigu sévère 2 (SRAS-CoV-2) est associée à une mortalité élevée dans les unités de soins intensifs (USI). Nous avons voulu décrire les caractéristiques cliniques et les issues des patients gravement atteints de la maladie à coronavirus 2019 (COVID-19) en contexte canadien.

MÉTHODES : Nous avons procédé à l'étude rétrospective d'une série de cas graves d'infection au SRAS-CoV-2 confirmée en laboratoire hospitalisés dans l'une des 6 USI du Vancouver métropolitain, en Colombie-Britannique (Canada), entre le 21 février et le 14 avril 2020. Les données démographiques, les rensei- gnements sur la prise en charge et les résultats ont été recueillis à partir des dossiers médicaux, électroniques ou non, des patients.

RÉSULTATS : Entre le 21 février et le 14 avril 2020, 117 patients ont été admis dans une USI avec un diagnostic confirmé de COVID-19. L'âge médian était de 69 ans (écart interquartile [EI] 60-75 ans); et 38 $(32,5 \%)$ étaient des femmes. Au moins une comorbidité était présente chez 86 patients $(73,5 \%)$. La ventilation mécanique a été nécessaire chez 74 patients (63,2\%). La durée de la ventilation mécanique a été de 13,5 jours (El 8-22 jours) dans l'ensemble et de 11 jours (II 6-16) chez les patients qui ont reçu leur congé de l'USI. Du tocilizumab a été administré à 4 patients et de l'hydroxychloroquine à 1 patient. En date du 5 mai 2020, 18 patients $(15,4 \%)$ étaient décédés, 12 (10,3\%) étaient toujours à l'USI, 16 (13,7\%) avaient obtenu leur congé de l'USI, mais restaient hospitalisés, et $71(60,7 \%)$ avaient pu retourner à la maison.

INTERPRÉTATION : Dans cette étude, la mortalité chez les patients gravement malades de la COVID-19 hospitalisés dans une USI a été moins élevée que chez les patients d'études précédentes. Ces résultats donnent à penser que le pronostic des cas graves de COVID-19 pourrait ne pas être aussi sombre que ce qui avait d'abord été rapporté.
$\mathbf{L}$

e coronavirus du syndrome respiratoire aigu sévère 2 (SRAS-CoV-2) est responsable de la maladie à coronavirus 2019 (COVID-19), qui a maintenant le statut de pandémie selon l'Organisation mondiale de la Santé1. Le taux d'infection et le nombre de décès dans le monde ont augmenté de façon exponentielle. Environ 35000 cas confirmés et plus de 1600 décès avaient été recensés au Canada en date du 21 avril 2020². En Colombie-Britannique, au 20 mai 2020, on dénombrait 2467 cas confirmés et 149 décès ${ }^{3}$. Par ailleurs, le nombre de nouveaux cas est en baisse depuis le début d'avril 2020. Plus de $85 \%$ des cas de COVID-19 en Colombie-Britannique ont été enregistrés dans la région métropolitaine de Vancouver ${ }^{3}$.
Les premières études en provenance de la Chine $^{4}$ et de l'Italie ${ }^{5}$ faisaient état de taux de mortalité allant de $26 \%$ à $62 \%$ chez les patients atteints d'une forme grave de COVID-19. Des études menées à Seattle ${ }^{6}$ et à New York ${ }^{7}$ ont quant à elles rapporté une mortalité globale de $23 \%$ à $50 \%$. Dans ces séries de cas, de $13 \%$ à $71 \%$ des patients étaient toujours aux soins intensifs au moment de publier, ce qui signifie que la mortalité réelle pourrait être plus élevée. On dispose actuellement d'un volume insuffisant de données canadiennes qui décrivent les cas graves de COVID-19 sont lacunaires, et il est indispensable d'arriver à mieux les caractériser afin de guider l'allocation des ressources et de comprendre la maladie dans notre contexte. Le but de 
notre série de cas multicentrique était de décrire les caractéristiques démographiques, la prise en charge et les résultats chez les patients atteints d'une forme grave de COVID-19 dans la région métropolitaine de Vancouver.

\section{Méthodes}

Nous avons étudié une série correspondant à tous les cas de COVID-19 hospitalisés dans une USI dans la région métropolitaine de Vancouver entre le 21 février et le 14 avril 2020, avec un suivi de l'issue de la maladie jusqu'au 5 mai 2020. Cette région sert environ 3 millions de Britanno-Colombiens (population provinciale de 4,9 millions d'habitants). Les hôpitaux inclus étaient l'Hôpital général de Vancouver (46 lits d'USI, centre de soins quaternaires), l'Hôpital Surrey Memorial (46 lits d'USI, centre de soins tertiaires), l'Hôpital Lions Gate (11 lits d'USI, hôpital communautaire), l'Hôpital St. Paul (15 lits d'USI, centre de soins tertiaires), l'Hôpital Royal Columbian (30 lits d'USI, centre de soins tertiaires) et l'Hôpital de Richmond (8 lits d'USI, hôpital communautaire). Toutes ces USI peuvent compter sur des médecins intensivistes, le ratio infirmières-patients y est d'environ 1:1,2, et les unités sont affiliées à l'Université de la Colombie-Britannique. Il s'agit d'unités mixtes qui prennent en charge les patients aussi bien médicaux que chirurgicaux. Dans le contexte de la pandémie, ces hôpitaux ont été désignés centres COVID-19 et, à ce titre, on y a transféré les patients ayant une forme grave de COVID-19 de leur circonscription hospitalière. L'admission à l'USI se faisait à la discrétion de l'intensiviste de garde, mais en général, on y admettait tous les patients présentant une infection par le SRASCoV-2 présumée ou confirmée ou nécessitant une quantité rapidement croissante d'oxygène, de l'oxygène par canule nasale à haut débit, une ventilation à pression positive non effractive, une ventilation mécanique ou des vasopresseurs. Tous les patients consécutifs ayant obtenu une confirmation en laboratoire de leur infection par le SRAS-CoV-2 qui ont été hospitalisés dans l'une de ces USI durant la période de l'étude ont été inclus.

La confirmation du SRAS-CoV-2 en laboratoire était définie par un résultat positif au test d'amplification en chaîne par polymérase $(\mathrm{PCR})$ en temps réel couplée à une transcription inverse sur des prélèvements de sécrétions du nez, du pharynx ou des voies respiratoires inférieures.

\section{Collecte des données}

Les données ont été extraites des dossiers médicaux des patients, électroniques ou non, dans chaque établissement par une équipe d'examinateurs cliniques professionnels, 2 intensivistes (A.R.M. et A.W.) et un assistant de recherche clinique; l'équipe a recueilli les données démographiques, les antécédents et les examens, les résultats d'analyses de laboratoire et les résultats cliniques des patients tout au long de chacune des hospitalisations. La gravité de la maladie a été établie selon le score APACHE II (Acute Physiology and Chronic Health Evaluation II) calculé à partir des données des 24 premières heures d'hospitalisation à l'USI ${ }^{8}$. Une évaluation du score SOFA (Sequential Organ Failure Assessment) ${ }^{9}$ pour le premier jour d'hospitalisation à l'USI a servi à préciser l'acuité de la maladie. Selon les paramètres, ce sont soit les résul- tats d'analyses de laboratoire de départ, soit les valeurs maximales atteintes au cours des 3 premiers jours du séjour à l'USI qui ont été retenues. Les paramètres de la ventilation mécanique ont été documentés après stabilisation, soit environ 24 heures après l'intubation. Les traitements reçus durant le séjour à l'USI ont été consignés, y compris les mesures d'appoint et l'administration d'agents pharmacologiques. Tous les examens et les traitements se faisaient à la discrétion des médecins traitants. Les données quotidiennes sur les hospitalisations et les séjours aux USI ont été fournies par les régies régionales de la santé Vancouver Coastal et Fraser. Les données sur la capacité des USI provenaient de la phase 1 de la projection du nombre de lits de soins intensifs requis pour les cas de COVID-19.

\section{Analyse statistique}

Des statistiques descriptives ont été utilisées pour résumer les caractéristiques démographiques, les caractéristiques cliniques, les résultats des analyses de laboratoire initiales à l'admission et, pour les 3 premiers jours à l'USI, la fréquence des interventions et des traitements, la prévalence d'admission à l'USI et les résultats cliniques. Les variables continues ont été présentées sous forme de moyennes et d'écarts-types ou de médianes et d'écarts interquartiles (EI), selon le cas. Les variables des catégories ont été présentées sous forme de nombres totaux et de pourcentages, à moins d'indication contraire. Aucune supposition n'a été faite pour les données manquantes. Toutes les analyses ont été effectuées à l'aide de Stata 15.2.

\section{Approbation éthique}

Le Comité d'éthique de la recherche clinique de l'Université de la Colombie-Britannique et le Comité d'éthique de la recherche de la Régie régionale de la santé Fraser ont approuvé cette étude. En raison de la nature rétrospective de l'étude et du risque minimal posé par celle-ci, l'obtention du consentement éclairé n'a pas été jugée nécessaire.

\section{Résultats}

Entre le 21 février et le 14 avril 2020, nous avons recensé 117 patients atteints d'une forme grave d'infection par le SRASCoV-2 confirmée en laboratoire, hospitalisés dans une USI de la région. Il n’y a eu aucune exclusion. La prévalence quotidienne des patients hospitalisés pour COVID-19, stratifiée en fonction de l'admission à l'USI, est présentée à la figure 1. Chaque jour, une proportion médiane de $40 \%$ (El 38\%-45\%) des patients hospitalisés a été admise à l'USI.

Des patients hospitalisés, 88 (75,2\%) arrivaient de leur domicile, 25 (21,4\%) d'un autre hôpital, et 4 (3,4\%) d'un établissement de soins de longue durée. Les caractéristiques démographiques et cliniques de départ sont présentées au tableau 1 et elles se comparent à celles de rapports précédents (annexe 1, accessible ici www.cmaj.ca/lookup/suppl/doi:10.1503/cmaj.200794/-/DC1). L'âge médian des patients était de 69 ans (EI 60-75 ans), avec une étendue de 23 à 92 ans. Sur l'ensemble, 38 patientes (32,5\%) étaient des femmes, et la durée moyenne des symptômes avant l'admission à l'USI était de 8 jours (écart-type 4,5). En tout, 


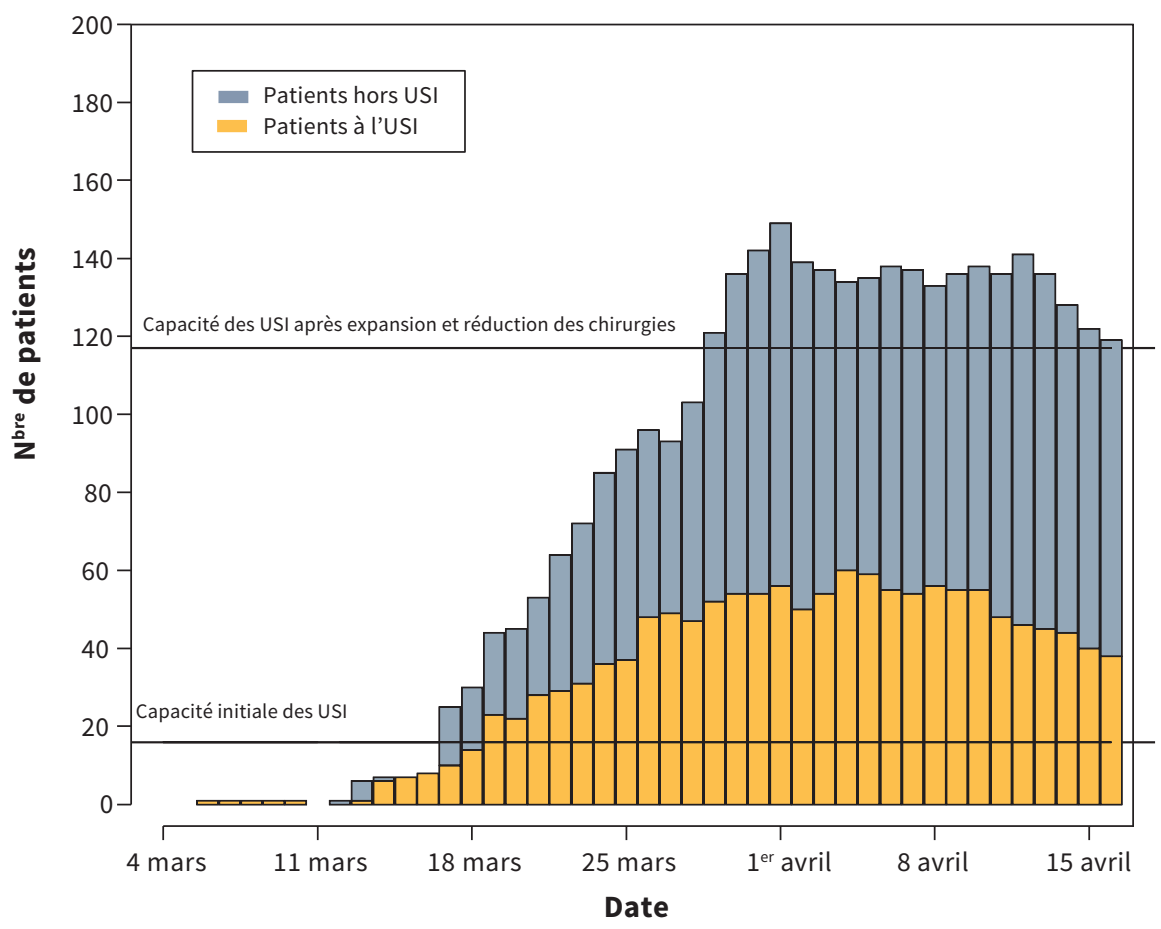

Figure 1 : Courbe épidémiologique de la prévalence quotidienne de patients hospitalisés pour maladie à coronavirus 2019 (COVID-19), stratifiée par admission à l'unité de soins intensifs (USI). La « capacité initiale des USI » fait référence au nombre de lits disponibles au-delà d'un taux d'occupation de base. La « capacité des USI après expansion et réduction des chirurgies » fait référence aux mesures spécifiques adoptées en raison de la COVID-19, soit l'augmentation du nombre de lits et les nouveaux lits accessibles en raison de l'annulation des chirurgies électives et non urgentes.

86 patients $(73,5 \%)$ étaient atteints d'au moins 1 comorbidité. Les comorbidités les plus fréquentes étaient l'hypertension (54 patients, 46,2\%), la dyslipidémie (43 patients, 36,8\%) et le diabète (36 patients, 30,8\%).

Les résultats des analyses de laboratoire au moment de l'admission à l'USI et au cours des 3 premiers jours à l'unité sont présentés au tableau 2. Une numération leucocytaire de départ supérieure à $10,0 \times 10^{9} / \mathrm{L}$ était présente chez 35 patients (29,9\%), et $9(7,7 \%)$ avaient des numérations inférieures à $4 \times 10^{9} / \mathrm{L}$. La lymphocytopénie était fréquente : 79 patients $(67,5 \%)$ avaient une numération lymphocytaire inférieure à $1,0 \times 10^{9} / \mathrm{L}$. En tout, 37 patients $(31,6 \%)$ avaient des taux de créatinine sérique de $106 \mu \mathrm{mol} / \mathrm{L}$ ou plus. Le lactate sérique initial était de $2,0 \mathrm{U} / \mathrm{L}$ ou plus chez 21 patients (17,9\%). En tout, 67 patients (57,2\%) avaient une concentration de D-dimères supérieure à $500 \mu \mathrm{g} / \mathrm{L}$ au cours des 3 premiers jours à l'USI, avec une médiane globale de 1560 (EI $740-4000) \mu \mathrm{g} / \mathrm{L}$. Dans l'ensemble, 32 patients $(27,4 \%)$ ont présenté un pic de troponine I supérieur à $26 \mathrm{ng} / \mathrm{L}$ ou un pic de troponine $\mathrm{T}$ supérieur à $0,02 \mathrm{ng} / \mathrm{L}$ au cours des 3 premiers jours à l'USI.

La fréquence des interventions et des traitements de soins intensifs est présentée au tableau 3. Les interventions les plus fréquentes ont été la ventilation mécanique (74 patients, 63,2\%), l'administration de vasopresseurs (65 patients, 55,6\%), le bloc neuromusculaire (50 patients, $42,7 \%$ ) et la pose de canules nasales à haut débit (43 patients, 36,8\%). Les valeurs médianes de $\mathrm{PaO}_{2}: \mathrm{FiO}_{2}$ (rapport entre la pression partielle de l'oxygène et la fraction d'oxygène inspirée) et de la compliance pulmonaire statique après 24 heures de ventilation mécanique étaient 180 ( $\mathrm{EI}$ 148-216) et 35 (El 31-44) $\mathrm{mL} \times \mathrm{cm} \mathrm{H}_{2} \mathrm{O}^{-1}$, respectivement. Aucun patient de notre étude n'a reçu de remdésivir, un patient $(0,9 \%)$ a reçu de l'hydroxychloroquine et 4 (3,4\%) ont reçu du tocilizumab.

En date du 5 mai 2020, en tout, 18 patients (15,4\%) étaient décédés, 12 (10,3\%) étaient toujours à l'USI, 16 (13,7\%) avaient reçu leur congé de I'USI, mais étaient toujours hospitalisés, et 71 $(60,7 \%)$ avaient pu retourner à la maison (tableau 3 , figure 2 ). Chez les 28 patients encore à l'hôpital, l'âge moyen était de 69 ans (EI 62-73), et la durée médiane du séjour à l'USI et à l'hôpital était de 29 jours (EI 18-33) et de 35 jours (EI 28-42), respectivement. La mortalité chez les patients sortis de I'USI (décès ou congé de l'USI) a été de 18 sur 105 (17,1\%). Chez les 18 patients décédés, l'âge médian était de 75 ans (intervalle 47-91), et 17 sur 18 (94,4\%) présentaient au moins une comorbidité. Les dossiers de 2 des 18 patients décédés portaient la mention «ne pas intuber».

Parmi les 74 patients qui ont eu besoin d'une ventilation mécanique, 12 (16,2\%) sont demeurés à l'USI, 13 (17,6\%) ont reçu leur congé de l'USI, mais sont restés hospitalisés, 34 (45,9\%) ont pu retourner à la maison, et $15(20,3 \%)$ sont décédés. Sur les 16 patients qui ont eu besoin d'un traitement continu de suppléance rénale, $6(37,5 \%)$ sont décédés, 7 (43,8\%) ont eu leur congé de l'USI et 3 (18,8\%) ont pu retourner à la maison. Les 16 (100\%) ont eu besoin de ventilation mécanique pendant leur séjour à l'USI. 
Tableau 1 : Caractéristiques démographiques et cliniques de base de 117 patients atteints d'une forme grave de COVID-19 hospitalisés dans des USI du Vancouver métropolitain, Colombie-Britannique

\section{Caractéristique}

Caractéristiques démographiques

Âge médian, ans (EI)

Sexe (féminin)

IMC médian (EI)†

Intervalle moyen entre le déclenchement des symptômes et l'hospitalisation à

I'USI, j ( \pm écart-type)

Score APACHE II médian (EI)†

Score SOFA à l'admission médian (EI)§

Sites participants

Quaternaire ou tertiaire A

Quaternaire ou tertiaire B

Quaternaire ou tertiaire C

Quaternaire ou tertiaire D

Communautaire A

Communautaire B

Comorbidités

Hypertension

Dyslipidémie

Diabète

Aucune

Maladie cardiovasculaire

Fumeur ou ex-fumeur

Maladie rénale chronique

Asthme

Maladie pulmonaire obstructive chronique

Cancer actif

Insuffisance cardiaque congestive

AVC hémorragique ou ischémique

Maladie hépatique chronique
$\mathbf{N}^{\text {bre }}(\%)$ de patients*

$69(60-75)$

$28(24-33)$

$8,0( \pm 4,5)$

$18(10-28)$

$6(2-11)$

$39(33,3)$

$29(24,8)$

$21(17,9)$

$9(7,7)$

$13(11,1)$

$6(5,1)$

$54(46,2)$

$43(36,8)$

$36(30,8)$

$31(26,5)$

$20(17,1)$

$16(13,7)$

$15(12,8)$

$14(12,0)$

$8(6,8)$

$7(6,0)$

$4(3,4)$

$4(3,4)$

$2(1,7)$

Remarque : $\mathrm{APACHE}=$ Acute Physiology and Chronic Health Evaluation, AVC = accident vasculaire cérébral, COVID-19= maladie à coronavirus 2019, EI = écart interquartile, IMC = indice de masse corporelle, SOFA = Sequential Organ Failure Assessment., USI = unité de soins intensifs.

*À moins d'indication contraire.

†L'IMC n’a été enregistré que pour les 74 patients placés sous ventilation mécanique. Les données étaient disponibles pour 55 d'entre eux (74,3\%).

łLe score APACHE II est une mesure de l'acuité de la maladie avec des scores pouvant varier de 0 à 71 . Un score plus élevé indique une maladie plus grave et est associé à une mortalité plus élevée. Les scores APACHE II étaient disponibles pour 95 patients (81,2\%).

$\S$ Le score SOFA est une mesure de l'acuité de la maladie avec des scores pouvant aller de 0 à 24 . Un score plus élevé indique une maladie plus grave et est associé à une mortalité plus élevée. Les scores SOFA au premier jour d'hospitalisation à l'USI étaient disponibles pour 115 patients $(98,3 \%)$

\section{Interprétation}

Dans cette série de cas graves d'infection par SRAS-CoV-2 confirmée en laboratoire hospitalisés dans l'une des 6 USI du Vancouver métropolitain, la mortalité globale a été nettement moindre que dans des études publiées précédemment, même si les caractéristiques de départ des patients étaient comparables, et malgré une proportion plus élevée de congés de l'hôpital. Les trois quarts des patients inclus dans notre étude ont reçu leur congé de l'USI, et $61 \%$ sont retournés à la maison. Contrairement à d'autres études, la mortalité n'a pas changé de façon marquée pour les patients soumis à une ventilation mécanique ou ayant reçu leur congé de l'USI.

Des séries de cas précédentes ont montré une mortalité élevée chez les patients atteints d'une forme grave de COVID-19 qui ont été hospitalisés. Dans une série de cas rétrospectifs 
Tableau 2 : Données de laboratoire à l'admission à l'USI et durant le séjour à l'USI pour 117 patients atteints

d'une forme grave de COVID-19

Variables

À l'admission à l'USI

Numération leucocytaire, $\times 10^{9} / \mathrm{L}$

Numération lymphocytaire, $\times 10^{9} / \mathrm{L}$

Numération plaquettaire, $\times 10^{9} / \mathrm{L}$

Créatinine sérique, $\mu \mathrm{mol} / \mathrm{L}$

Bilirubine totale, $\mu \mathrm{mol} / \mathrm{L}$

Bilirubine directe, $\mu \mathrm{mol} / \mathrm{L}$

Aspartate aminotransférase, $\mathrm{mU} / \mathrm{mL}$

Alanine aminotransférase, $\mathrm{mU} / \mathrm{mL}$

Lactate, $\mathrm{mmol} / \mathrm{L}$

Au cours des 3 premiers jours à l'USI

Numération plaquettaire la plus basse, $\times 10^{9} / \mathrm{L}$

Pic de créatinine sérique, $\mu \mathrm{mol} / \mathrm{L}$

Concentration de bilirubine totale la plus élevée, $\mu \mathrm{mol} / \mathrm{L}$

Concentration de bilirubine directe la plus élevée, $\mu \mathrm{mol} / \mathrm{L}$

Taux le plus élevé de D-dimères, $\mu \mathrm{g} / \mathrm{L}$

Concentration de ferritine la plus élevée, $\mathrm{ng} / \mathrm{mL}$

Concentration de protéine C-réactive la plus élevée, $\mathrm{mg} / \mathrm{L}$

Concentration de créatine kinase la plus élevée, U/L

Concentration de troponine I la plus élevée, ng/L

Concentration de troponine T la plus élevée, $\mathrm{ng} / \mathrm{L}$

Données de laboratoire, médianes (EI)

Références

$\begin{array}{cc}8,1(5,9-10,8) & 3,5-12,0 \\ 0,8(0,5-1) & 1,0-3,0 \\ 215(161-271,5) & 150-400 \\ 86(70-114) & 50-110 \\ 9(6-14) & 3-22 \\ 3,5(2-7) & 0-5 \\ 63,5(38-99) & 18-40 \\ 44(27-88) & 17-63 \\ 1,5(1,1-1,9) & 1-1,8\end{array}$

$190(155-250)$

$150-400$

101 (79-148)

50-110

$10(7-15)$

3-22

$5(3-9)$

$0-5$

$1560(740-4000)$

$0-229$

1125 (637-2058)

$15-400$

$148(87-232)$

$0-10$

$119(49-255)$

$25-200$

$15(7-40)$

$<26$

$0,02(0,02-0,08)$

Remarque : COVID-19 = maladie à coronavirus 2019, EI = écart interquartile, USI = unité de soins intensifs.

regroupant 1591 patients hospitalisés dans une USI de Lombardie, en Italie, la mortalité globale était de $26 \% 5$. Fait à noter : la majorité de la cohorte était toujours à l'USI au moment de la publication. La mortalité chez les patients sortis de l'USI (décès ou congé) était de $61 \%$. Dans une série de 24 cas de Seattle, la mortalité globale était de $50 \%$, mais elle était de $57 \%$ chez ceux qui étaient sortis de I'USI'. Dans une série de 52 cas gravement atteints admis dans une USI de Wuhan, en Chine, la mortalité globale était de $62 \%$, et elle était de $80 \%$ chez ceux qui étaient sortis de I'USI ${ }^{4}$. Une série de cas de New York a fait état d'une mortalité globale de $23 \%$ chez les patients ayant séjourné à I'USI'. Le congé de I'USI n'ayant pas été rapporté, la mortalité chez les patients sortis de l'USI n'a pas pu être calculée. La mortalité chez les patients gravement atteints sortis de l'hôpital (décès ou congé) de cette série était de $78 \%{ }^{7}$. Un atout de l'étude actuelle est qu'il s'agit d'une étude multicentrique regroupant toutes les USI de la région métropolitaine de Vancouver qui ont traité des patients pour la COVID-19, ce qui inclut des USI de centres de soins quaternaires, tertiaires et communautaires.

Les patients de notre étude présentaient des similitudes avec les séries de cas de Lombardie, de Seattle, de New York et de Wuhan pour ce qui est de l'âge, des comorbidités et de la gravité déclarée de la maladie. La gravité de la maladie chez les patients semble aussi comparable à celle d'autres études au plan des covariables mesurées (p. ex., $\mathrm{PaO}_{2}: \mathrm{FiO}_{2}$, compliance, scores APACHE II et SOFA). Les patients de notre étude ont moins eu besoin de ventilation mécanique effractive $(63,2 \%)$ que les patients des études de Lombardie (88\%), de Seattle (75\%) et de New York $(90 \%)$, mais en ont plus eu besoin que la cohorte de Wuhan (42\%). L'utilisation de la ventilation en position ventrale $(17,9 \%)$ chez nos patients a été similaire à ce qu'on a observé en Lombardie (27\%), à Seattle (28\%) et à Wuhan (12\%).

Dans la région métropolitaine de Vancouver, nous avons initialement hésité à utiliser la canule nasale à haut débit au début de la pandémie en raison d'inquiétudes concernant le risque d'aérosolisation et de propagation du SRAS-CoV-2. Toutefois, après une revue multidisciplinaire ${ }^{10}$, et compte tenu que les canules nasales à haut débit ont été associées à une mortalité moindre dans les cas d'insuffisance respiratoire hypoxémique ${ }^{11}$, nous avons décidé de les utiliser chez les patients atteints de la COVID-19, moyennant l'application de mesures de protection contre la transmission par aérosols.

Malgré les différences observées entre les patients et les interventions de soins intensifs de ces études, on ignore si elles expliquent à elles seules la mortalité nettement moindre que nous rapportons. Selon notre hypothèse, ces résultats encourageants 
Tableau 3 : Traitements intensifs et résultats cliniques chez 117 patients atteints d'une

forme grave de COVID-19

\section{Variable}

Traitements intensifs

Ventilation mécanique

Vasopresseurs

Bloc neuromusculaire

Canule nasale à haut débit

Ventilation en position ventrale

Traitement continu de suppléance rénale

Ventilation à pression positive non effractive

Vasodilatateurs pulmonaires par inhalation

Oxygénation par membrane extracorporelle

Paramètres médians de ventilation mécanique $24 \mathrm{~h}$ après l'intubation

(EI)†

Pression de plateau, $\mathrm{cm} \mathrm{H}_{2} \mathrm{O}$

$22(20-24)$

Pression positive en fin d'expiration, $\mathrm{Cm} \mathrm{H}_{2} \mathrm{O}$

$12(10-14)$

$50(40-60)$

$\mathrm{FiO}_{2}, \%$

$\mathrm{PaO}_{2}: \mathrm{FiO}_{2}$

$180(148-216)$

Volume courant, $\mathrm{mL} / \mathrm{kg}$

Compliance, $\mathrm{mL} \times \mathrm{cm} \mathrm{H}_{2} \mathrm{O}^{-1}$

400 (350-450)

$35(31-44)$

Traitement pharmacologique

Corticostéroïdes

Tocilizumab

$28(23,9)$

$4(3,4)$

Hydroxychloroquine

Remdésivir

Issues

Décès à l'USI

$1(0,9)$

$0(0,0)$

$18(15,4)$

$12(10,3)$

$16(13,7)$

$71(60,7)$

$10(8,5)$

$6(5,1)$

$9(5-21)$

$7(3-16)$

$6(3-11)$

$12(7-18)$

Décès

Durée médiane du séjour hospitalier, j (EI)

Globale

$18(11-30)$

$18(10-32)$

$15(8-22)$

$13(8-18)$

Décès

Durée médiane de la ventilation mécanique, $\mathrm{j}$ (EI)

Globale, $n=74$

$13,5(8-22)$

Extubation, $n=50$

$11,5(6-18)$

$11(6-16)$

$8(5-13)$

$13(10-20)$

Patients ayant eu leur congé de l'hôpital, $n=34$

Décès, $n=15$

Remarque : $\mathrm{COVID}-19$ = maladie à coronavirus 2019, $\mathrm{EI}=$ écart interquartile, $\mathrm{FiO}_{2}=$ fraction de l'oxygène inspiré, $\mathrm{PaO}_{2}=$ pression partielle de l'oxygène, USI = unité de soins intensifs.

*À moins d'indication contraire

tLes paramètres complets de la ventilation mécanique 24 heures après son instauration étaient disponibles pour 48 patients sur 74 $(64,9 \%)$ soumis à la ventilation mécanique. 


\section{A) Âge $<55$ ans}

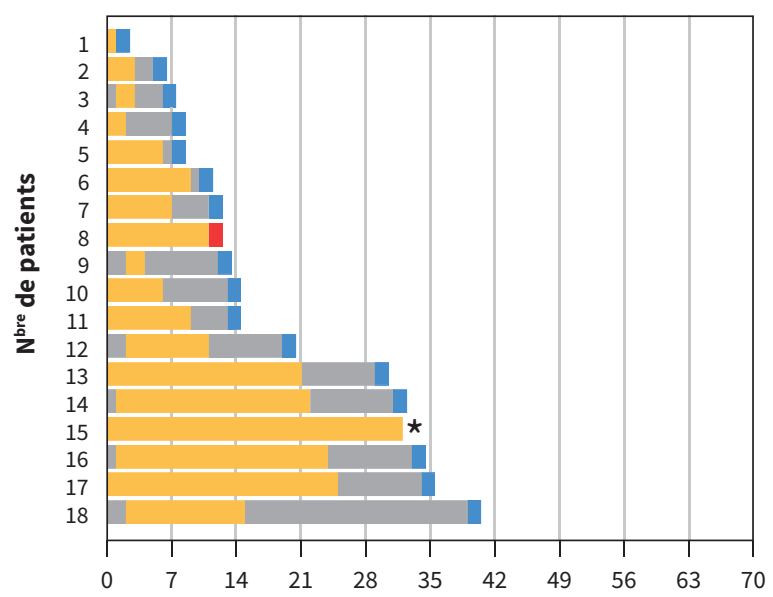

C) Âge 65-69 ans

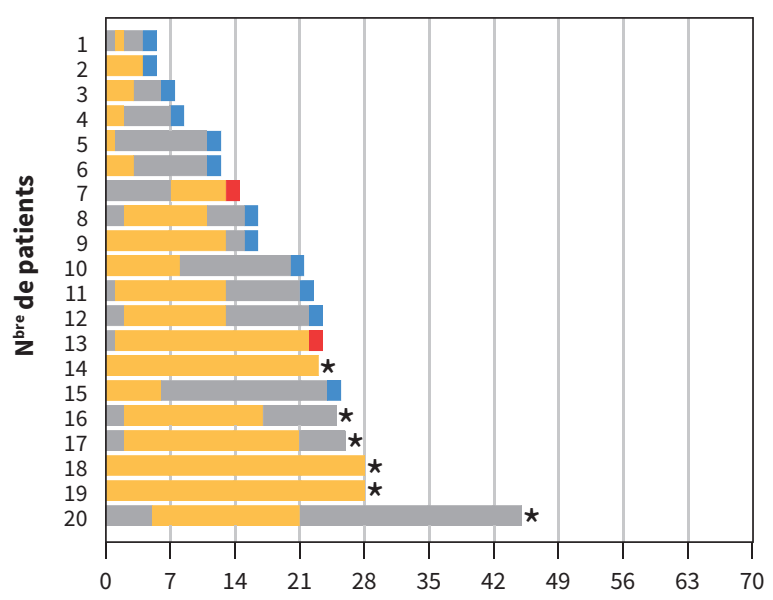

E) Âge 75-79 ans

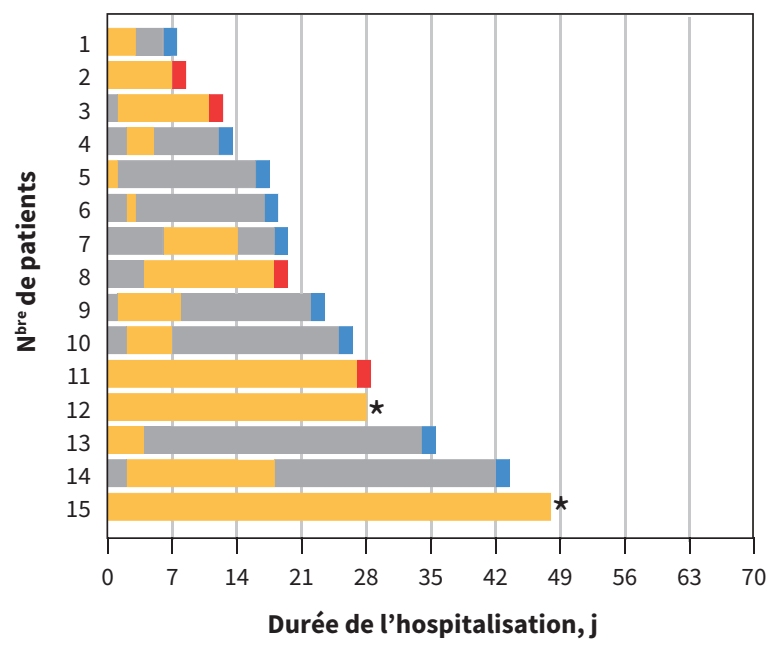

\section{B) Âge 55-64 ans}

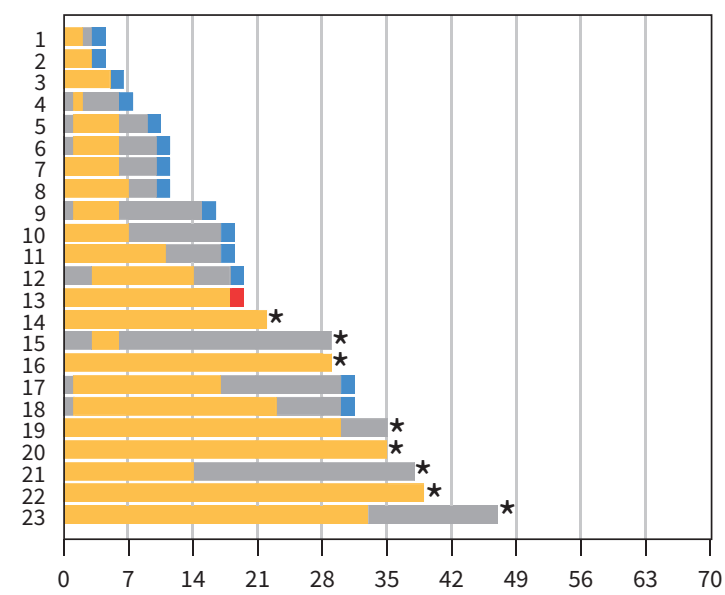

D) Âge 70-75 ans

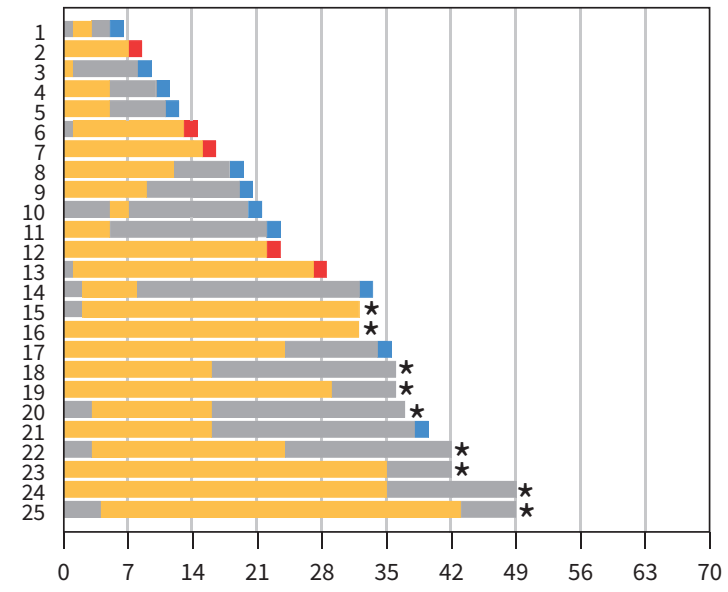

F) Âge $>80$ ans

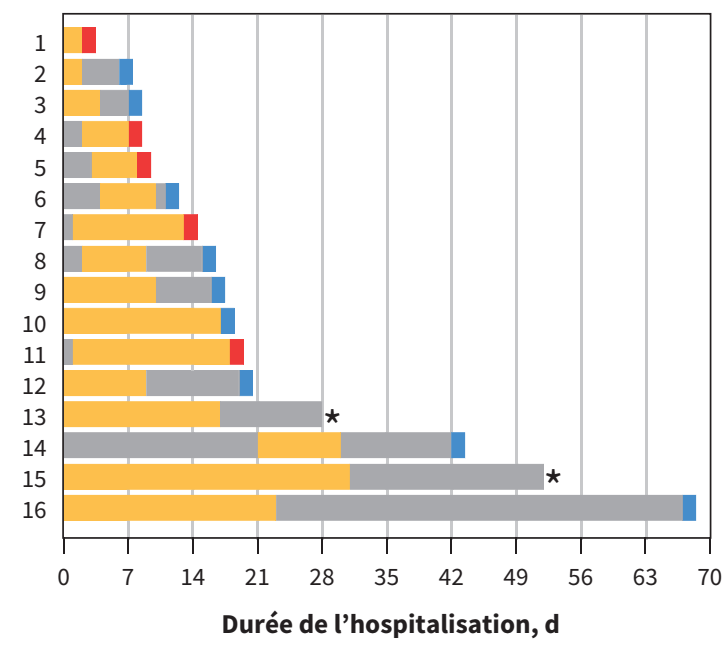

\begin{tabular}{|l|lll}
\hline Hôpital USI $\quad$ Congé hospitalier $\quad$ Décès $\quad$ Arrêt de la collecte des données \\
\hline
\end{tabular}

Figure 2 : Graphique à barres horizontales montrant le nombre de jours d'hospitalisation chez les patients stratifiés selon l'âge (A-F). *Les données censurées font référence aux données des patients toujours hospitalisés (aux soins intensifs ou non) au moment de l'arrêt de la collecte des données à la fin de l'étude. USI = unité de soins intensifs. 
pourraient être attribuables à une réponse plus vaste à l'échelle systémique qui a permis d'éviter un afflux insurmontable de patients gravement atteints de la COVID-19 dans nos hôpitaux et nos USI. Chaque jour, environ $40 \%$ de notre population COVID-19 totale hospitalisée était admise à l'USI, ce qui témoigne de la disponibilité des lits. Même si les auteurs n'ont pas rapporté les pourcentages quotidiens, l'étude de New York récemment publiée mentionnait que $22 \%$ de sa population totale de patients hospitalisés avait séjourné à l'USI à un moment ou à un autre ${ }^{7}$. Comme nous dans la région métropolitaine de Vancouver, d'autres régions ont aussi appliqué leurs interventions à l'échelle systémique. Or, ces réponses ont été adoptées après que les hôpitaux avaient déjà connu un afflux de patients atteints de COVID-1912,13. La prévention d'une surcharge de nos USI leur a permis de conserver les ressources nécessaires pour offrir des soins intensifs optimaux aux patients atteints de la COVID-19. Les ratios normaux travailleurs de la santé:patients ont été maintenus, l'approvisionnement en équipement de protection individuelle était suffisant et les travailleurs de la santé n'ont pas eu à prodiguer des soins en dehors de leur champ de pratique normal. Une priorisation de l'allocation des ressources n'a pas été requise, et tous les patients dont les objectifs de soins concordaient avec la mission des USI ont bénéficié des soins intensifs.

La disponibilité des ressources en soins intensifs a été assurée par la mise en œuvre de mesures de santé publique visant à réduire l'incidence de la COVID-19 et par une planification provinciale-régionale ayant pour but d'augmenter la capacité des USI. En plus de l'éloignement sanitaire, d'autres mesures de santé publique ont été appliquées de manière séquentielle parallèlement au début de la relâche scolaire du printemps, le 14 mars $^{3}$. Les interdictions suivantes ont été promulguées le 16 mars : grands rassemblements de plus de 50 personnes, entrée de ressortissants étrangers sur le territoire canadien et arrivée par avion d'individus symptomatiques au Canada ${ }^{3}$. À ce moment, les vols internationaux de retour ont été limité à 4 aéroports nationaux ${ }^{3}$. D'autres mesures de santé publique ont été adoptées, notamment : déclaration de l'urgence sanitaire par les autorités provinciales et auto-isolement obligatoire des voyageurs sur ordre de la santé publique à partir du 17 mars, déclaration d'un état d'urgence provincial et application de restrictions concernant les services de bars et de restaurants le 18 mars, fermeture de la frontière entre les États-Unis et le Canada pour les déplacements non essentiels le 20 mars, et fermeture des commerces de services personnels le $21 \mathrm{mars}^{3}$. Ensemble, ces mesures ont permis d'aplatir la courbe de prévalence épidémiologique en ColombieBritannique, illustrée à la figure 1 .

Simultanément, la capacité des soins intensifs a été substantiellement accrue. Le 16 mars, les chirurgies non urgentes et électives ont été annulées, afin de libérer des lits de soins intensifs. Cela a aussi libéré des lits d'unité de soins intensifs de chirurgie cardiaque et de salles de réveil, qui ont été ajoutés aux lits de soins intensifs déjà disponibles dans les centres COVID-19 du Vancouver métropolitain. La capacité des USI avant la pandémie, aux taux d'occupation antérieurs, et la nouvelle capacité des USI après l'expansion et la réduction des chirurgies sont présentées à la figure 1 .
Même en l'absence de pandémie, l'augmentation de la pression exercée sur la capacité d'accueil des USI peut entraîner une hausse de la mortalité perhospitalière ${ }^{14}$, particulièrement chez les grands malades ${ }^{15}$. En outre, les régions comptant des taux élevés de patients atteints de COVID-19 présentaient une mortalité non liée à la COVID-19 plus élevée, conséquence possible de la surcharge du système de santé ${ }^{16,17}$. Grâce aux mesures de santé publique et à l'accroissement de la capacité des USI, nous avons pu répondre aux besoins de tous les patients atteints de la COVID-19 dans nos hôpitaux et nos unités de soins intensifs. Une autre importante observation de notre étude est que ce faible taux de mortalité a été obtenu sans utilisation ciblée de certains médicaments pour la COVID-19 ${ }^{18}$. Nos conclusions ne démentent pas l'efficacité des traitements pharmacologiques proposés pour les patients atteints d'une forme grave de COVID-19, mais elles indiquent qu'il est possible d'obtenir d'excellents résultats cliniques avec des soins intensifs de soutien de haut niveau et accessibles, indépendamment des traitements pharmacologiques ciblés.

\section{Limites de l'étude}

Cette étude comporte plusieurs limites importantes. Étant donné qu'il s'agit d'une série de cas, il est impossible de déterminer si des liens causaux pourraient expliquer les résultats rapportés. Toute association entre, d'une part, la capacité et les mesures appliquées à l'échelle systémique, et de l'autre, les résultats observés, est spéculative. La durée du suivi est relativement brève par rapport au cours de la maladie, et les données rapportées sur la mortalité et la durée des séjours dans cette étude pourraient changer. Enfin, les résultats ne sont pas disponibles pour tous les patients, et certains étaient encore à l'USI ou à l'hôpital à la fin de la collecte des données.

\section{Conclusion}

Dans cette série de cas graves d'infection par le SRAS-CoV-2 confirmée en laboratoire hospitalisés dans des USI de la région métropolitaine de Vancouver, au Canada, la mortalité globale était de $15 \%$. Ce taux est inférieur aux taux rapportés précédemment, malgré la faible utilisation de traitements pharmacologiques ciblés, ce qui pourrait être le reflet de la gérabilité de la clientèle, rendue possible par l'application de mesures par les autorités sanitaires régionales et par l'augmentation de la capacité des USI.

\section{Références}

1. WHO director-general's opening remarks at the media briefing on COVID-1911 March 2020. Geneva: World Health Organization; 11 mars 2020. Accessible ici : www.who.int/dg/speeches/detail/who-director-general-s-opening -remarks-at-the-media-briefing-on-covid-19---11-march-2020 (consulté le 21 avril 2020).

2. Coronavirus disease 2019 (COVID-2019) situation report - 92. Geneva: World Health Organization; 21 avril 2020. Accessible ici : www.who.int/emergencies/ diseases/novel-coronavirus-2019/situation-reports (consulté le 21 avril 2020).

3. BC COVID-19 dashboard. Vancouver: BC Centre for Disease Control; mis à jour le 20 mai 2020. Accessible ici : www.bccdc.ca/health-info/diseases-conditions/ covid-19/data (consulté le 20 mai 2020).

4. Yang $X, Y u Y, X u$ J, et al. Clinical course and outcomes of critically ill patients with SARS-CoV-2 pneumonia in Wuhan, China: a single-centered, retrospective, observational study. Lancet Respir Med 2020;8:475-81. 
5. Grasselli G, Zangrillo A, Zanella A, et al. Baseline characteristics and outcomes of 1591 patients infected with SARS-CoV-2 admitted to ICUs of the Lombardy Region, Italy. JAMA 6 avril 2020 [Cyberpublication avant impression]. doi: 10.1001/jama.2020.5394.

6. Bhatraju PK, Ghassemieh BJ, Nichols M, et al. COVID-19 in critically ill patients in the Seattle region - case series. N Engl J Med 30 mars 2020 [Cyberpublication avant impression]. doi: 10.1056/NEJMoa2004500.

7. Richardson S, Hirsch JS, Narasinhan M, et al. Presenting characteristics, comorbidities, and outcomes among 5700 patients hospitalized with COVID-19 in the New York City Area. JAMA 22 avril 2020 [Cyberpublication avant impression]. doi: 10.1001/jama.2020.6775.

8. Knaus WA, Draper EA, Wagner DP, et al. APACHE II - a severity of disease classification system. Crit Care Med 1985;13:818-29.

9. Vincent JL, Moreno R, Takala J, et al. The SOFA (Sepsis-related Organ Failure Assessment) score to describe organ dysfunction/failure. Intensive Care Med 1996;22:707-10.

10. High-flow oxygen during the COVID-19 pandemic. Vancouver: British Columbia Center for Disease Control; mis à jour le 15 avril 2020. Accessible ici : www. bccdc.ca/Health-Professionals-Site/Documents/COVID19_HighFlowOxygen Recommendations.pdf (consulté le 7 mai 2020).

11. Frat JP, Thille AW, Mercat A, et al. High-flow oxygen through nasal cannula in acute hypoxemic respiratory failure. N Engl J Med 2015;372:2185-96.

12. Grasselli G, Pesenti A, Cecconi M. Critical care utilization for the COVID-19 outbreak in Lombardy, Italy: early experience and forecast during an emergency response. JAMA 13 mars 2020 [Cyberpublication avant impression]. doi: 10.1001/jama.2020.4031. [Cyberpublication avant impression].

13. Wu Z, McGoogan JM. Characteristics of and important lessons from the coronavirus disease 2019 (COVID-19) outbreak in China: summary of a report of 72314 cases from the Chinese Center for Disease Control and Prevention. JAMA 2020; 24 février [Cyberpublication avant impression]. doi: 10.1001/jama.2020.2648.

14. Fergusson NA, Ahkioon S, Nagarajan M, et al. Association of intensive care unit occupancy during admission and inpatient mortality: a retrospective cohort study. Can J Anaesth 2020;67:213-24.

15. Wilcox ME, Harrison D, Patel A, et al. Higher ICU capacity strain is associated with increased acute mortality in closed ICUs. Crit Care Med 2020;48:709-16.

16. Lazzerini M, Barbi E, Apicella A, et al. Delayed access or provision of care in Italy resulting from fear of COVID-19. Lancet Child Adolesc Health. 2020;4:e1011. doi: 10.1016/S2352-4642(20)30108-5.

17. Covid 19's death toll appears higher than official figures suggest. The Economist 4 avril 2020. Accessible ici : www.economist.com/graphic-detail/2020/04/03/ covid-19s-death-toll-appears-higher-than-official-figures-suggest (consulté le 21 avril 2020).

18. Clinical Reference Group Recommendation: therapies for COVID-19. Vancouver: British Columbia Center for Disease Control; 2020:1-3. [mis à jour le 12 avril 2020]. Accessible ici : www.bccdc.ca/Health-Professionals Site/Documents/Recommendation_Unproven_Therapies_COVID-19.pdf (consulté le 21 avril 2020).

\section{Intérêts concurrents : Aucun intérêt concurrent déclaré}

Cet article a été révisé par des pairs.

Affiliations : Division de médecine de soins intensifs, Département de médecine (Mitra, Wormsbecker, Foster, Karpov, Haljan, Chittock, Kanji, Sekhon, Griesdale), Université de la Colombie-Britannique, Vancouver, C.-B.; Division de médecine de soins intensifs, Département de médecine (Mitra, Haljan), Hôpital Surrey Memorial, Surrey, C.-B.; programme de doctorat en médecine (Fergusson), Université de la Colombie-Britannique; Qualité et sécurité des patients (Fergusson, Lloyd-Smith), régie régionale de la santé Vancouver Coastal, Vancouver, C.-B.; Division de médecine de soins intensifs, Département de médecine (Wormsbecker), Hôpital Royal Columbian, New Westminster, C.-B.; Division de médecine de soins intensifs, Département des infirmières praticiennes (Crowe), Hôpital Surrey Memorial, Surrey, C.-B.; Programme de soins intensifs (Chittock, Kanji, Sekhon, Griesdale), Hôpital général de Vancouver; Départements d'anesthésiologie, de pharmacologie et de thérapeutique (Griesdale), Université de la Colombie-Britannique; Centre d'épidémiologie et d'évaluation clinique (Griesdale), Institut de recherche de la régie régionale de la santé Vancouver Coastal, Vancouver, C.-B.

Collaborateurs : Donald Griesdale, Anish Mitra et Nicholas Fergusson ont eu pleinement accès à toutes les données de l'étude et assument la responsabilité de l'intégrité des données et de l'exactitude de leur analyse. Anish Mitra et Nicholas Fergusson ont contribué à parts égales à ces travaux à titre de co-auteurs. Anish Mitra, Nicholas Fergusson, Dean Chittock et Donald Griesdale ont contribué à la conception et au protocole. Tous les auteurs ont recueilli, analysé ou interprété les données. Anish Mitra, Nicholas Fergusson et Donald Griesdale ont rédigé la pre- mière version du manuscrit. Anish Mitra, Nicholas Fergusson et Donald Griesdale ont procédé aux analyses statistiques. Tous les auteurs ont participé à la révision critique du manuscrit; ils ont donné leur approbation définitive pour la version destinée à être publiée et assument l'entière responsabilité de tous les aspects du travail.

Financement : Donald Griesdale bénéficie d'une subvention de la Michael Smith Foundation for Health Research à titre de professionnel de la santé-investigateur. Mypinder Sekhon bénéficie d'une bourse de l'Institut de recherche de la régie régionale de la santé Vancouver Coastal à titre de clinicien chercheur.

Partage des données : Un ensemble de données dépersonnalisées est hébergé sur un serveur sécurisé à la régie régionale de la santé Vancouver Coastal. Des ententes sur le partage des données en empêchent la publication. Les demandes de données peuvent être accordées moyennant une entente appropriée à cet effet qui impliquerait les autorités des régies régionales de la santé Vancouver Coastal et Fraser. Le code d'analyse des données (Stata 15.2) est disponible sur demande.

Remerciements : Les auteurs remercient Steven Reynolds, Neilson McLean et Demetrious Sirounis pour leur soutien à l'accès aux données. Ils remercient en outre le personnel infirmier et le personnel des unités de soins intensifs pour les soins prodigués et leur dévouement envers les patients. En terminant, ils remercient leurs patients pour leur participation à cette étude. Aucun des sujets n'a été rémunéré pour son rôle dans l'étude.

Accepté : 12 mai 2020

Correspondance : Donald Griesdale, donald.griesdale@ubc.ca 